\title{
Dominant LMNA mutations can cause combined muscular dystrophy and peripheral neuropathy
}

\author{
S Benedetti, E Bertini, S lannaccone, C Angelini, M Trisciani, D Toniolo, B Sferrazza, P Carrera, \\ G Comi, M' Ferrari, A Quattrini, S C Previtali
}

J Neurol Neurosurg Psychiatry 2005;76:1019-1021. doi: 10.1136/jnnp.2004.046110

\begin{abstract}
The coexistence of neurogenic and myogenic features in scapuloperoneal syndrome is rarely ascribed to a single gene. Defects in the nuclear envelope protein lamin A/C, encoded by the LMNA gene, have been shown to be associated with a variety of disorders affecting mainly the muscular and adipose tissues and, more recently, with autosomal recessive Charcot-Marie-Tooth type 2 neuropathy. This report is about a patient presenting features of myopathy and neuropathy due to a dominant LMNA mutation, suggesting that the peripheral nerve might be affected in primary LMNA myopathy. Our observations further support the marked intrafamilial and interfamilial phenotypic heterogeneity associated with lamin A/C defects.
\end{abstract}

l: nterpretation of muscle weakness and wasting with scapuloperoneal distribution has been controversial due to the coexistence of myopathic and neurogenic features. Besides the clear myopathic (such as Emery-Dreifuss or facioscapulohumeral dystrophy) or neurogenic forms linked to chromosome 12q, some patients show a combined phenotype, with primarily myopathic features in the upper limbs and neurogenic features in the lower limbs. ${ }^{12}$ Diagnosis of these combined myopathic-neurogenic syndromes has benefited from the recent advances in molecular genetics. In fact, it has been shown that single gene mutations can affect both muscles and nerves, such as in LAMA2 deficiency (mutation of laminin $\alpha 2$ gene), mitochondrial disorders, glycogenosis type III, ${ }^{3}$ or, more recently, congenital hypomyelinating neuropathy. ${ }^{4}$

Mutations in the lamin $\mathrm{A} / \mathrm{C}$ gene $(L M N A)$, encoding a nuclear intermediate filament protein, are responsible for different disorders, including autosomal Emery-Dreifuss muscular dystrophy, limb-girdle muscular dystrophy type 1B, dilated cardiomyopathy with conduction system disease, autosomal recessive Charcot-Marie-Tooth (CMT) type 2B1, familial partial lipodystrophy (Dunnigan type), mandibuloacral dysplasia, and Hutchinson-Gilford and atypical Werner (progeria) syndromes. ${ }^{5}$ Although LMNA mutations have been associated with such heterogeneous phenotypes, only one case of combined myopathic and neurogenic features has been reported so far. ${ }^{6}$ Here we describe another patient with clear coexistence of muscular dystrophy and axonal neuropathy associated with dominantly inherited LMNA mutations.

\section{METHODS}

Biopsy samples were processed following standard protocols as previously described. ${ }^{7}$ Genomic DNA was extracted from peripheral blood and screened for LMNA mutations by denaturing high performance liquid chromatography (DHPLC) and sequencing, also described previously. ${ }^{8}$ GJBl and MPZ coding exons with flanking intronic sequences were amplified from genomic DNA and directly sequenced. Primer sequences and polymerase chain reaction (PCR) conditions are available upon request.

\section{CASE REPORT}

The patient (DE) is a 14 year old boy from a nonconsanguineous family from southern Italy (fig lA). He achieved his early developmental milestones normally. When he was 6 years old, it was noticed that he ran clumsily with frequent stumbling. He developed progressive weakness and a tendency to walk on his heels and tiptoes. He underwent surgery to correct pes equinovarus. By the age of 13 years he was mostly wheelchair bound. Neurological examination revealed bilateral steppage and a waddling gait, which was only possible with external help. Muscle atrophy and weakness were severe distally and moderate proximally in the lower limbs, whereas muscle trophism and mild weakness were present in the upper limbs. Tendon reflexes

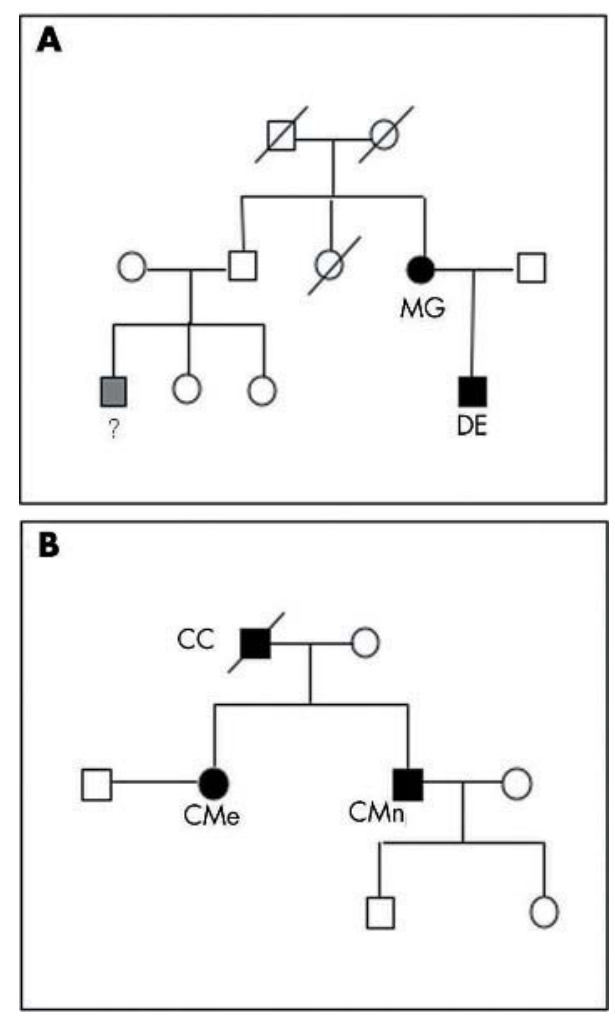

Figure 1 (A) Pedigree of the Italian family carrying the heterozygous p. R571C mutation; the relative with the uncertain phenotype is indicated by a question mark. (B) Pedigree of the Filipino family carrying the heterozygous frameshift c.864_867del mutation. 
were absent in all four limbs. Superficial and discriminative sensations were nearly normal whereas proprioceptive sensation was mildly impaired (lack of position sense at both toes) and Romberg's sign was positive. Peripheral nerves were not thickened and intellect was normal. He was observed to have fat accumulation in the face and neck with "buffalo hump" distribution.

His serum creatine kinase was five times above normal; lactate dehydrogenase, the transaminases, and triglyceride were mildly elevated, and initial signs of glucose intolerance were present. Other routine laboratory tests, including screening for dysimmune/inflammatory disorders, electrocardiography, and echocardiography, were normal. No mutations were detected in SMN1, hexosaminidase, and two genes responsible for axonal CMT (MPZ and GJB1). Neurophysiological evaluation showed severely reduced amplitudes of the compound muscle action potentials (cMAPs) of the right peroneal nerve and moderate reduction of velocity ( $200 \mu \mathrm{V}$; velocity $38.0 \mathrm{~m} / \mathrm{s})$. The cMAPs of the left peroneal nerve were not recordable and the amplitude of
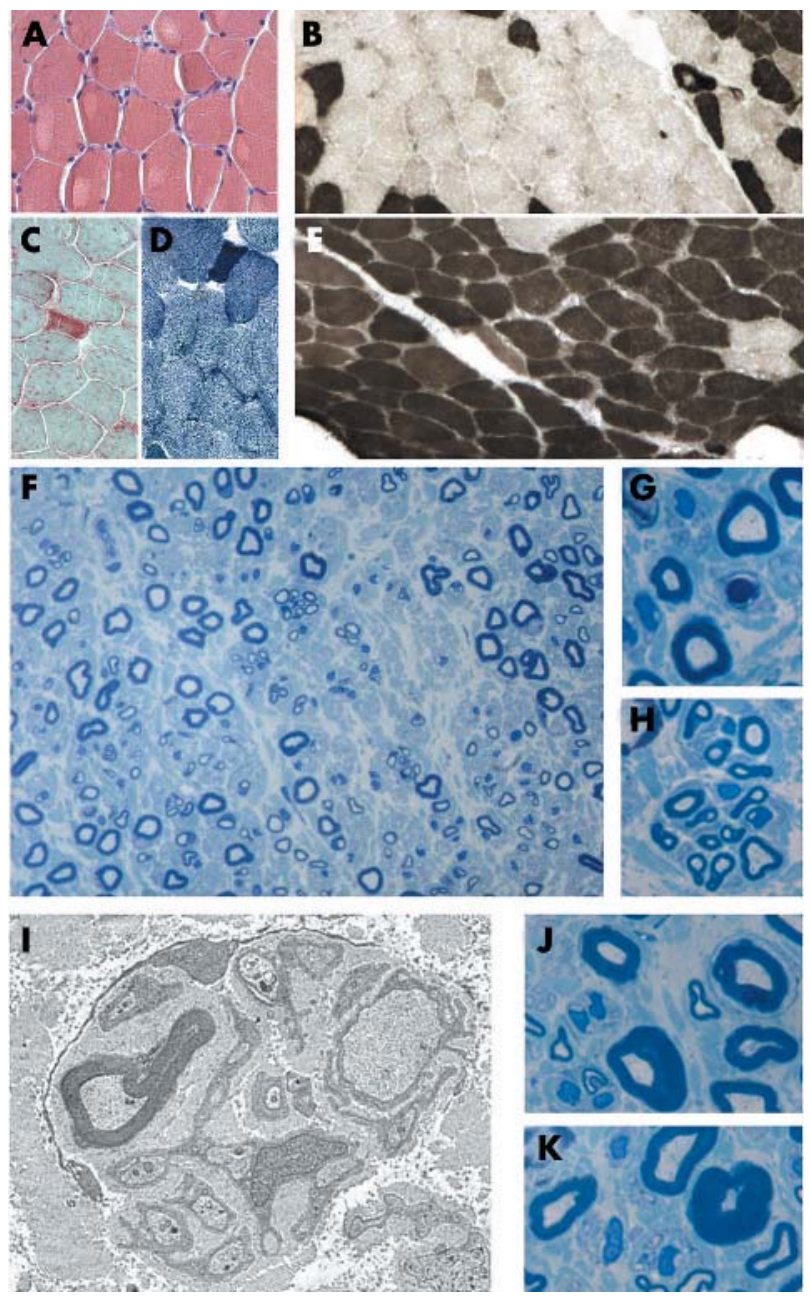

Figure 2 ( $A-E)$ Muscle and $(F-K)$ sural nerve biopsies from patient $D E$. Increased variability in fibre size is seen with (A) haematoxylin and eosin, (D) nicotinamide adenine dinucleotide-tetrazolium reductase, (NADH-TR), and (B, E) ATPase pH 4.6 staining; (C) necrotic fibre seen with acid phosphatase staining; (D) small angulated "dark" fibre seen with NADH-TR staining; and $(B, E)$ fibre-type grouping seen with ATPase $\mathrm{pH} 4.6$ staining. Semithin sections show (F) reduced number of myelinated fibres, $(G)$ acute axonal degeneration, and $(H)$ clusters of regeneration. Rare onion bulbs are seen with (I) electron microscopy and (J) semithin section. (K) Few fibres with abnormally thick myelin are seen. Magnification: $\times 50(A-E), \times 200(F), \times 500(G, H, J, K)$, and $\times 3000(I)$. sensory nerve action potentials (SAPs) in the sural nerves was reduced $(3.8 \mu \mathrm{V}$; velocity $46.9 \mathrm{~m} / \mathrm{s})$. No conduction blocks were observed. cMAPs, SAPs, and velocities were in the normal range in the upper limbs. Electromyography showed acute (insertion activity, fibrillation, and positive sharp waves) and chronic (large motor unit potentials) signs of denervation in the distal muscles of the lower limbs (tibialis anterior and gastrocnemius). Minimal denervation and myopathic signs were observed in the proximal muscles of the four limbs (deltoid, biceps brachialis, and vastus medialis).

A muscle biopsy from the vastus lateralis showed myopathic and neurogenic features including atrophic fibres, few necrotic fibres, increased connective tissue, and fibretype grouping (fig 2A-E). A sural nerve biopsy showed moderate loss of primarily large and medium sized myelinated fibres, some degenerating fibres, and numerous clusters of regeneration (fig $2 \mathrm{~F}-\mathrm{H}$ ). Rare myelinated fibres revealed abnormally thick myelin sheath or small onion bulbs (fig 2I-K). Neither inflammatory cells nor abnormalities of the nerve blood vessels were observed.

The patient's 54 year old mother (MG) had no complaints of motor or sensory deficits. Neurological examination was normal. She showed fat accumulation in the neck and face. Neurophysiological studies showed mild reduction of cMAP of the peroneal nerve $(1.6 \mathrm{mV}$, velocity $49.1 \mathrm{~m} / \mathrm{s})$ and SAP of the sural nerves $(3.0 \mu \mathrm{V}$, velocity $53.8 \mathrm{~m} / \mathrm{s})$. Her upper limbs showed normal amplitudes and velocities. Another relative complaining of myopathic symptoms could not be further characterised (fig lA). The patient's father underwent clinical and neurophysiological evaluation and did not show any signs of peripheral neuropathy.

\section{Second case report (different LMNA mutation)}

A 37 year old woman (CMe) from a non-consanguineous family originating from the Philippines (fig 1B) was asymptomatic until the age of 25 years, when she required a pacemaker implantation because of severe atrioventricular block. After the age of 33 years, she developed minor difficulties in walking and fatigability. Neurological examination showed weakness limited to the proximal muscles of the four limbs, moderate muscle atrophy, mild hypotonia, and areflexia. Serum creatine kinase and the other routine laboratory tests were normal. Antiacetylcholine receptor antibodies were absent. Electromyography showed myopathic signs in the proximal muscles. Electroneurography did not reveal significant abnormalities. A muscle biopsy (quadriceps) showed mild myopathic and neurogenic features, including increased connective tissue, atrophic rounded fibres, some small angulated fibres hyperreactive with staining for oxidative enzymes (nicotinamide adenine


Figure 3 Muscle biopsy from patient CMe. (A) Haematoxylin and eosin stain shows variability in fibre size; small angulated "dark" fibers are shown with staining for (B) nicotinamide adenine dinucleotidetetrazolium reductase (NADH-TR) and (C) succinic dehydrogenase (SDH); (D, E) ATPase pH 4.6 show fibre-type grouping. Magnification: $\times 50(A-E)$. 
dinucleotide-tetrazolium reductase, NADH-TR; succinic dehydrogenase, SDH), and fibre-type grouping (fig 3). Dystrophin, sarcoglycans, and emerin were normally expressed. No mutations were detected in the MPZ and GJBl genes.

Her 35 year old brother (CMn) had no complaints of any motor or sensory deficits. Neurological examination revealed moderate amyotrophy and mild proximal weakness in the lower limbs, hyporeflexia, and syndactyly of the fourth and fifth fingers of the right foot. Their father, CC, died suddenly while asleep at 40 years of age. Since adolescence, he had had difficulties in walking and frequent falls.

\section{MOLECULAR GENETIC STUDIES}

Molecular analysis in both MG (mother) and DE (son), revealed a heterozygous c.1711C $>\mathrm{T}$ mutation, causing a p.R57lC substitution in a conserved region of lamin $\mathrm{C}$ specific carboxyl-terminal domain. This alteration was not present in 180 healthy controls. Interestingly, a similar mutation affecting the same codon (p.R571S) has been previously described in association with atrial fibrillation and dilated cardiomyopathy and no signs of skeletal muscle involvement. ${ }^{9}$

Molecular analysis of the LMNA gene in CMe and CMn showed a heterozygous frameshift deletion in exon 5 (c.864_867del), leading to the formation of a premature stop codon after 198 amino acids (p.H289RfsX189).

\section{DISCUSSION}

Mutations in the LMNA gene have been associated with myopathy (Emery-Dreifuss or limb-girdle muscular dystrophy) or neuropathy (autosomal recessive CMT2), along with other cardiac, endocrinological, and skeletal disorders. Here we report the clear coexistence of scapuloperoneal myopathy and predominantly axonal neuropathy in a patient with LMNA mutation. Muscle and nerve involvement are demonstrated at the clinical, neurophysiological, and histological levels. Interestingly, we also report a second family, carrying a different LMNA mutation, with manifest limb-girdle myopathy and histological findings of clear type grouping suggesting denervation-reinnervation, although no neurophysiological signs of peripheral neuropathy were present. We think that these findings might be indicative of a subclinical neuropathy that may become manifest in the course of the disease.

Our results suggest that the peripheral nerve compartment is affected in patients with primary LMNA myopathy. The association of myopathy and neuropathy with heterozygous LMNA defects has been recently described. ${ }^{6}$ However, here we report for the first time the histological evidence of a combined neuropathic and myopathic phenotype associated with a dominant LMNA alteration. This strengthens the hypothesis of a possible common aetiology for the muscle and nerve pathologies in our patients. The presence of a peripheral neuropathy should therefore be considered in patients carrying LMNA mutations.

Unravelling a neuropathic phenotype may also be important from the perspective of genetic correction of lamin deficiency in muscle. In fact, this intervention may not completely revert the clinical phenotype as demonstrated in animal models of LAMA2 myopathy, ${ }^{10}$ another condition with associated muscle dystrophy and peripheral neuropathy due to deficiency of laminin $\alpha 2 .^{11}$

In previous reports, a peripheral neuropathy associated with LMNA mutations was restricted to a homozygous p.R298C substitution ${ }^{12}$; a dominant missense p.E33D mutation has been described only recently. ${ }^{6}$ Here, we identify another heterozygous mutation of the LMNA gene (p.R57lC) associated with a CMT2 neuropathy, showing that similar phenotypes can be due to alterations in different regions of the protein. Notably, substitution of the same R57l codon was previously reported in a case of dilated cardiomyopathy with conduction defects. ${ }^{9}$ In our study, the mutation was found in two individuals from the same family harbouring clearly different phenotypes-overt neuropathy and myopathy in the child and subclinical peripheral nerve involvement in the mother-with similar subcutaneous fat redistribution. These observations confirm the remarkable clinical and allelic heterogeneity of LMNA related disorders and the difficulty in establishing any genotype-phenotype correlation.

\section{ACKNOWLEDGEMENTS}

The authors are indebted to S Grassi, F Corea, G Dina, and I Menditto.

\section{Authors' affiliations}

S Benedetti, M Ferrari, Laboratory of Clinical Molecular Biology, Diagnostica e Ricerca San Raffaele, Milano, Italy

E Bertini, Unit of Molecular Medicine, Ospedale Bambino Gesù, Roma, Italy

S lannaccone, B Sferrazza, G Comi, A Quattrini, S C Previtali, Department of Neurology, IRCCS San Raffaele Scientific Institute, Milano, Italy

C Angelini, Department of Neurosciences, University of Padova, Padova, Italy

M Trisciani, P Carrera, M Ferrari, Unit for Genomics for Human Disease Diagnosis, IRCCS San Raffaele Scientific Institute, Milano, Italy D Toniolo, Dibit, IRCCS San Raffaele Scientific Institute, Milano, Italy

Grant support from Italian Telethon (SCP GGP030193; CA no. GTF02009); FISM (SCP 2002/R/42).

Competing interests: none declared

Patients' consent was obtained

Correspondence to: Stefano C. Previtali, Department of Neurology, San Raffaele Scientific Institute, Via Olgettina 60, 20132 Milano, Italy; previtali.stefano@hsr.it

Received 21 May 2004

Revised version received 9 September 2004

Accepted 13 October 2004

\section{REFERENCES}

1 Walton J, Gardner-Medwin D. Progressive muscular dystrophy and the myotonic disorders. In: Walton J, ed. Disorders of voluntary muscles 3rd edn. Edinburgh: Churchill Livingstone, 1974:561-81,

2 Harding A. Inherited neuronal atrophy and degeneration predominantly of lower motor neurons. In: Dyck P, Thomas P, Griffin J, et al. Peripheral neuropathy, 3rd edn. Philadelphia: WB Saunders Co, 1993:1059-61.

3 Katirii B, Kaminski H, Preston D, et al. Neuromuscular disorders in clinical practice. Boston: Butterworth Heinemann, 2002:1432.

4 Szigeti K, Saifi G, Armstrong D, et al. Disturbance of muscle fiber differentiation in congenital hypomyelinating neuropathy caused by a novel myelin protein zero mutation. Ann Neurol 2003;54:398-402.

5 Mounkes L, Kozlov S, Burke B, et al. The laminopathies: nuclear structure meets disease. Curr Opin Genet Dev 2003;13:223-30.

6 Goizet C, Ben Yaou R, Demay L, et al. A new mutation of the lamin A/C gene leading to autosomal dominant axonal neuropathy, muscular dystrophy, cardiac disease, and leuconychia. J Med Genet 2004;41:e29.

7 Quattrini A, Previtali S, Feltri ML, et al. b4 integrin and other Schwann cell markers in axonal neuropathy. Glia 1996;17:294-306.

8 Vytopil M, Benedetti S, Ricci E, et al. Mutation analysis of the lamin A/C gene (LMNA) among patients with different cardiomuscular phenotypes. J Med Genet 2003;40:e132.

9 Fatkin D, MacRae C, Sasaki T, et al. Missense mutations in the rod domain of the lamin A/C gene as causes of dilated cardiomyopathy and conductionsystem disease. N Engl J Med 1999;341:1715-24.

10 Kuang $\mathrm{W}, \mathrm{Xu} \mathrm{H}$, Vachon $\mathrm{P}$, et al. Merosin-deficient congenital muscular dystrophy. J Clin Invest 1998;102:844-52.

11 Shorer Z, Philpot J, Muntoni F, et al. Demyelinating peripheral neuropathy in merosin-deficient congenital muscular dystrophy. J Child Neurol 1995; 10:472-5.

12 De Sandre-Giovannoli A, Chaouch M, Kozlov S, et al. Homozygous defects in LMNA encoding lamin A/C nuclear-envelope proteins, cause autosomal recessive axonal neuropathy in human (Charcot-Marie-Tooth disorder type 2) and mouse. Am J Hum Genet 2002;70:726-36. 